DOI: http://doi.org/10.4038/sljp.v22i1.8077

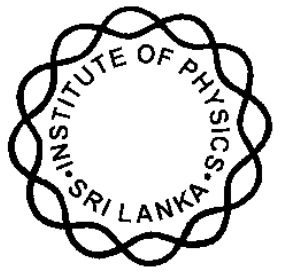

INSTITUTE OF PHYSICS - SRI LANKA

Research Article

\title{
Exfoliated graphite as electrochemical double layer capacitor electrode: Electrochemical impedance study
}

\author{
D.S.K. Rajaguru, K.P. Vidanapathirana*, K.S. Perera \\ Polymer Electronics Research Group, Department of Electronics, Wayamba University of \\ Sri Lanka, Kuliyapitiya, Sri Lanka
}

\begin{abstract}
Exfoliated graphite (EG) is one of the best alternatives for graphene in the electrochemical devices since graphene needed a cost-effective mass production to reach the commercialization. Liquid phase exfoliation has been identified as an inexpensive, ecofriendly method for high quality EG production. Sri Lankan natural graphite and commercially available graphite were used for exfoliation and the converted EG samples were used to fabricate electrochemical double layer capacitors (EDLCs). They were characterized using Electrochemical Impedance Spectroscopy (EIS). EG prepared by Sri Lankan natural graphite showed higher specific capacitance values compared with commercial graphite EG. Thereby, the value of Sri Lankan graphite over commercial graphite was emphasized.
\end{abstract}

Keywords: Electrochemical Double Layer Capacitor, Exfoliation, Exfoliated Graphite, Electrochemical Impedance Spectroscopy

* Corresponding author:kamalpv@,wyb.ac.lk

(DD https://orcid.org/0000-0003-0676-7627 


\section{INTRODUCTION}

Electrochemical Double Layer Capacitors (EDLCs) have been widely used as an energy storage device to fulfill the energy requirement ${ }^{1}$. EDLCs have higher power density and longer cyclic life than rechargeable batteries ${ }^{2}$. Therefore, many research works have been carried out to improve the performances. Their efforts mainly focus on improving the properties of electrode materials ${ }^{3}$.

Nowadays, EDLCs have been developed using various types of carbonaceous materials such as activated carbon, carbon nanotubes, carbon aerogels, carbon nanofibers and graphite due to ease of accessibility, low cost, non-toxicity, high chemical stability and wide temperature range $e^{4-8}$. Based on these materials, many approaches have been tested to increase the Specific Surface Area (SSA) of the electrode material ${ }^{9}$.

Since its discovery in 2004, graphene (one atom thickness-sp ${ }^{2}$ hybridized-two-dimensional carbon) has gained a lot of attention of global scientists ${ }^{10}$. Graphene has an extraordinarily high SSA up to $2630 \mathrm{~m}^{2} / \mathrm{g}^{11}$. Even though graphene proved to be an ideal electrode material which has remarkable electrochemical properties, its industrial applications were restricted. Deficiency of large-scale production of graphene and high production cost are the main reasons for this drawback. Graphite is composed of individual graphene sheets and it is the most inexpensive and easily obtained carbonaceous material. Therefore, various methodologies were carried out using graphite to achieve the requirements of a highperformance electrode material.

'Exfoliation' is a process where graphite gets separated into graphene layers. The degree of exfoliation or the graphene yield completely relies on the exfoliation method. Several methods have been identified throughout the past years. A most reasonable, inexpensive and ecofriendly method is the aqueous surfactant mediated liquid phase exfoliation ${ }^{12}$. This method avoids using toxic and expensive solvents, thus giving an easy and practical approach. EG is low dense graphitic carbon consists of few graphene layers with a high temperature resistance ${ }^{13}$. In addition to that, EG provides a porous carbon network that can act as an active material in the EDLC electrode.

Therefore, this study aims to use Sri Lankan natural graphite as electrode material in EDLCs while compromising its value over commercially available graphite. Electrochemical 
Impedance Spectroscopy (EIS) was used to investigate the capacitive properties of the fabricated EG based EDLCs.

\section{EXPERIMENTAL}

\subsection{Materials}

Sri Lankan natural graphite samples $\left(\mathrm{G}_{1}\right.$ - particle size $40 \mu \mathrm{m}, \mathrm{G}_{2}$ - particle size 10-15 $\left.\mu \mathrm{m}\right)$ were obtained from Bogala Graphite Lanka Ltd, Bogala, Sri Lanka. Commercial graphite $\left(\mathrm{G}_{3}\right.$ - particle size $\left.<20 \mu \mathrm{m}\right)$, sodium dodecyl benzene sulfonate (SDBS), polyvinilidine fluoride (PVDF), zinc trifluoromethanesulfonate (ZnTF), ethylene carbonate (EC), propylene carbonate (PC) and acetone were purchased from Sigma Aldrich and used without further purification.

\subsection{Preparationof exfoliated graphite electrodes}

Each graphite sample $\left(\mathrm{G}_{1}, \mathrm{G}_{2}, \mathrm{G}_{3}\right)$ were exfoliated in aq. SDBS solution $\left(0.1 \mathrm{mg} \mathrm{ml}^{-1}\right)$ using ultrasonic homogenizer (Athena ATP 150) for 45 minutes and filtered separately. Another set of graphite was sonicated in acetone prior to the exfoliation. The filtered exfoliated graphite samples were then dried and deposited onto fluorine-doped tin oxide (FTO) glasses as the electrodes. Acetone was used as the solvent for coating purpose. Area of the electrode was $1 \mathrm{~cm}^{2}$.

\subsection{Preparation of gel polymer electrolyte (GPE)}

Required amounts of PVDF, EC, PC and ZnTF were stirred well and heated. The hot mixture was pressed in between two glass plates to get a bubble-free thin film and was allowed to $\operatorname{dry}^{14}$.

\subsection{Fabrication and characterization of EDLCs}

Laboratory scale EDLCs were assembled using the exfoliated graphite electrodes and the prepared GPE. The EDLCs with different exfoliated graphite electrodes $\left(\mathrm{G}_{1}\right.$, pre-sonicated $\mathrm{G}_{1}, \mathrm{G}_{2}$, pre-sonicated $\mathrm{G}_{2}, \mathrm{G}_{3}$, pre-sonicated $\mathrm{G}_{3}$ ) were tested with Electrochemical Impedance Spectroscopy (EIS) technique in the frequency range $400 \mathrm{kHz}-10 \mathrm{mHz}$ using an impedance analyser (Metrohm M101). 


\section{RESULTS AND DISCUSSION}

EIS is related with impedance data which is one of the key characterization techniques use to study electrochemical devices. It reveals the properties of the electrolyte and the electrodes of an EDLC via Nyquist plots at different frequencies. Bulk electrolyte properties are observed in the high frequency range. Mid frequency range stands for the electrodeelectrolyte interface as well as the electrode properties. Low frequency range highlights capacitive features. Figure 1 demonstrates the Nyquist plots obtained for different graphite samples.

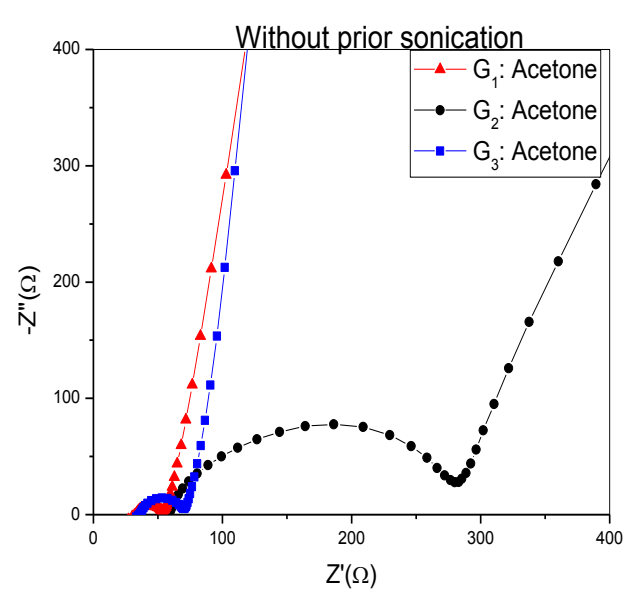

(a)

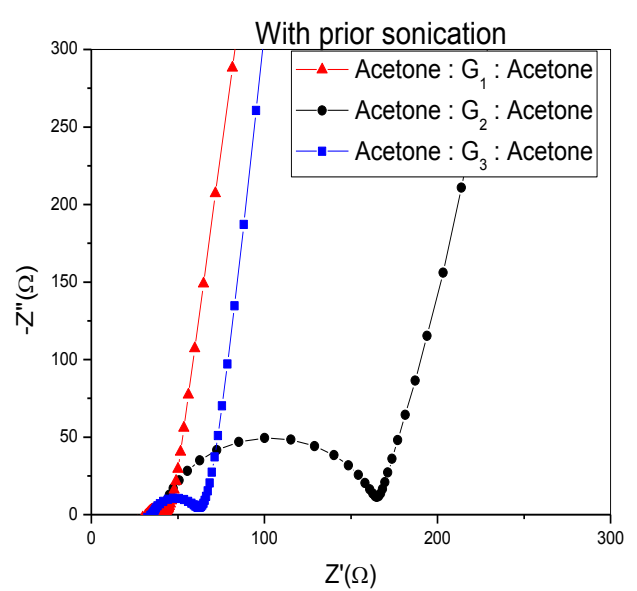

(b)

Figure 1: Comparison of Nyquist plots of different EG electrodes [(a) without prior sonication (b) with prior sonication in acetone] in the frequency range $400 \mathrm{kHz}$ to $10 \mathrm{mHz}$.

A typical Nyquist plot of an EDLC consists of two semicircles followed by a vertical line. First semicircle at the high frequency region gives an idea about the bulk electrolyte properties. X-axis intercept in the highest frequency region corresponds to bulk electrolyte resistance. Semicircle in the middle frequency region arises due to the charge transfer resistance (ionic and/or electronic). Straight line in the low frequency region represents capacitive features.

All the characteristics at mid and low frequencies were clearly observed for the three graphite samples in figure 1. Main differences are the diameter of semicircle and the $\mathrm{x}$-intercepts where the non-vertical lines appear. Smaller radius/diameter implies low charge transfer resistance ${ }^{15}$. Main reason for this variation is the pore size of the electrodes. When pores in the electrode material are larger there is more accessibility for ions to the electrode, giving low ionic resistance. Size of semicircle radius increases as $\mathrm{G}_{1}<\mathrm{G}_{3}<\mathrm{G}_{2}$. This may be due to the decrease in pore size of $E G$ in the order of $\mathrm{G}_{1}>\mathrm{G}_{3}>\mathrm{G}_{2}$. In the low frequency region, $a$ 
huge increase in the imaginary part of the impedance can be seen. This corresponds to the capacitive behavior connected with the adsorption of ions to the porous EG network. The vertical spike getting more parallel to the imaginary impedance axis indicating pure capacitive behavior ${ }^{16}$. Exfoliation process decreases the number of graphene layers stack together and thus give few layered graphite. Therefore, the surface area is higher in EG than in graphite.

When the sonication was done particle size will be reduced while the mixture is allowed to be uniform in composition. Decrease of the EG particle size due to the prior sonication permits to see the difference in the semicircle in Nyquist plots and the vertical lines getting more parallel to the imaginary axis.

Figure 2 (a), (b) shows the Bode plots of variation of real part of the complex capacitance $\left(C^{\prime}\right)$ with frequency for the different EG electrodes. Bode plots provide a convenient method to study the variation of capacitance with frequency. The capacitance values obtained for the EG electrodes at the lowest frequency, $0.01 \mathrm{~Hz}$ are summarized in table $1 . \mathrm{G}_{2}$ sample achieved the highest capacitance among the three.

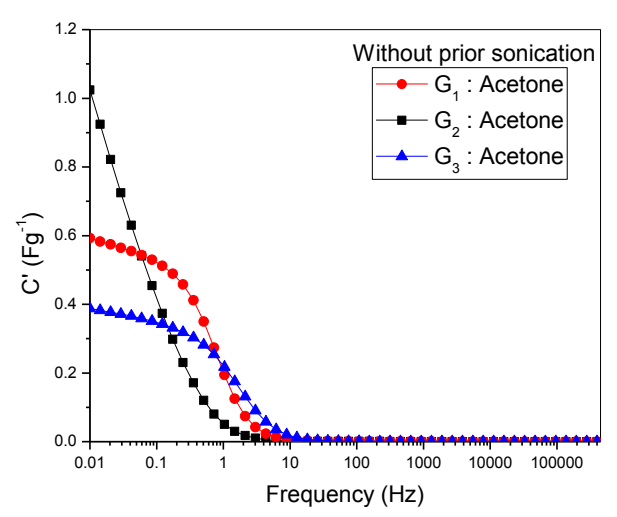

(a)

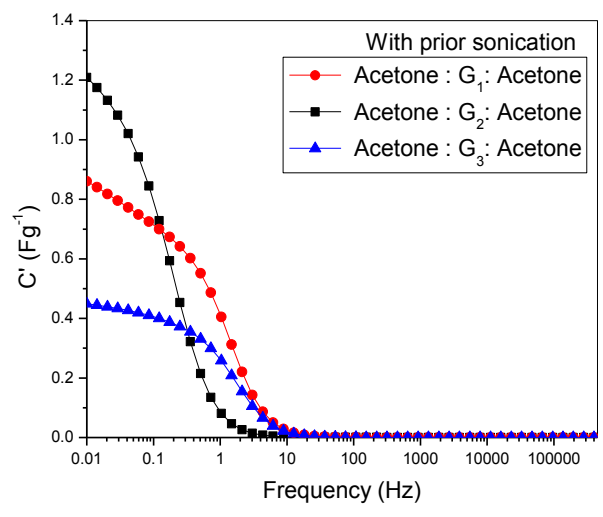

(b)

Figure 2: Comparison of Bode plots, $\mathrm{C}^{/}$versus frequency (in logarithmic scale) of different EG electrodes (a) without prior sonication (b) with prior sonication. 
Table 1: $\mathrm{C}^{\prime}, f_{0}$ and $\tau_{0}$ values obtained for the exfoliated graphite electrodes

\begin{tabular}{|c|c|c|c|c|}
\hline \multicolumn{2}{|c|}{ Electrode type } & $\mathbf{C}^{\prime}\left(\mathbf{F ~ g}^{-1}\right)$ & $f_{0}(\mathrm{~Hz})$ & $\tau_{0}(\mathbf{s})$ \\
\hline \multirow{3}{*}{ 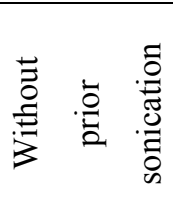 } & $\mathrm{G}_{2}:$ Acetone & 1.02 & 0.02 & 7.79 \\
\hline & $\mathrm{G}_{1}:$ Acetone & 0.59 & 0.73 & 0.22 \\
\hline & $\mathrm{G}_{3}:$ Acetone & 0.39 & 1.49 & 0.11 \\
\hline \multirow{3}{*}{ 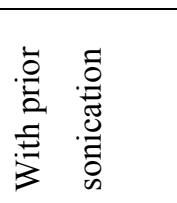 } & Acetone : $\mathrm{G}_{2}:$ Acetone & 1.21 & 0.17 & 0.91 \\
\hline & Acetone : $\mathrm{G}_{1}:$ Acetone & 0.86 & 1.49 & 0.11 \\
\hline & Acetone : $\mathrm{G}_{3}$ : Acetone & 0.45 & 1.49 & 0.11 \\
\hline
\end{tabular}

When frequency increases from $0.01 \mathrm{~Hz}$ to $1 \mathrm{~Hz}$, a drastic drop of $\mathrm{C}^{\prime}$ can be seen in the $\mathrm{G}_{2}$ sample. But for the other two, 'rapid decrease' appears only after $1 \mathrm{~Hz}$ and beyond $10 \mathrm{~Hz}$ the capacitance becomes insignificant, where the EDLCs act as a pure resistor ${ }^{17}$.

EG samples with prior sonication showed almost similar shapes except for the increase in $\mathrm{C}^{\prime}$ values. Although there is an increase in $\mathrm{C}^{\prime}$, the degree of increase is not the same for all. EG sample $\mathrm{G}_{2}$ showed about $18.6 \%$ increase and $\mathrm{G}_{1}$ EG showed about $45.8 \%$ increase while $\mathrm{G}_{3}$ showed only about $15.4 \%$ in the capacitance with prior sonication compared to the electrodes without prior sonication.

Additional information can be obtained by plotting the imaginary part of the complex capacitance $\left(\mathrm{C}^{/ /}\right)$versus frequency (Figure 3$)$.

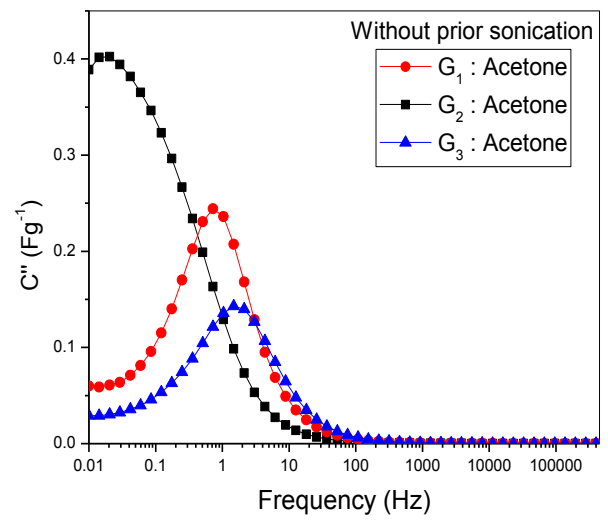

(a)

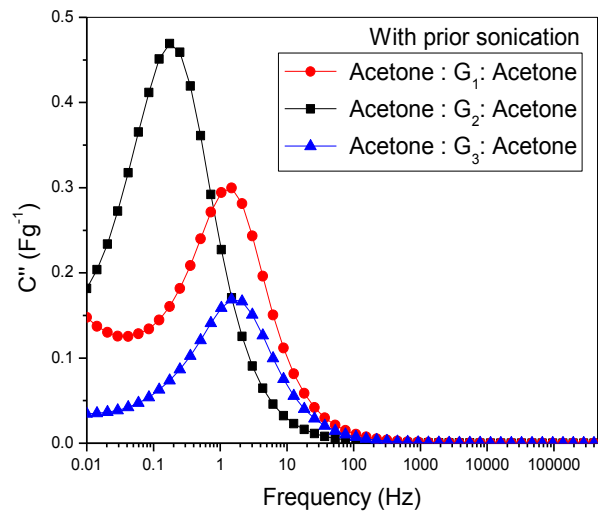

(b)

Figure 3: Comparison of Bode plots, $\mathrm{C}^{/ /}$versus frequency (in logarithmic scale) of different EG electrodes (a) without prior sonication (b) with prior sonication. 
The frequency, $f_{0}$ where the maximum of the curve occurred is a characteristic feature for each system. This $f_{0}$ can be identified as the point where the EDLC goes from purely resistive to purely capacitive when the frequency is changed from higher to lower value ${ }^{18}$. Relaxation time constant, $\tau_{0}$ is a quantitative measurement of how fast the EDLC can be charged and discharged reversibly and it indicates the time taken by the EDLC to reach half of its saturated capacitance ${ }^{17}$. Therefore, it can be simply defined as the time required to deliver the stored energy and power efficiently. This time constant can be deduced from $f_{0}$, using equation (1).

$$
\tau_{0}=\frac{1}{2 \pi f_{0}}
$$

$f_{0}$ values and the corresponding $\tau_{0}$ values were summarized in table 1 . The porosity of the EG electrodes may responsible for the fast ion transfer and thus giving very low time constants. Smaller $\tau_{0}$ value means a higher rate of accumulation and release of charges which is an essential requirement for an efficient EDLC.

With the prior sonication, Bode plot curves were shifted towards higher frequencies as in figure 3, indicating $\tau_{0}$ values to be smaller. For the EG sample $\mathrm{G}_{2}$, change of $\tau_{0}$ value with the prior sonication is very significant. This may be due to the open up of more pores with the sonication which enhances the charge movement. For the $E G$ sample $\mathrm{G}_{1}$, this change was moderate but for the EG sample $\mathrm{G}_{3}$, value was unchanged may be due to saturation where the time constant cannot surpass the minimum time limit.

\section{CONCLUSION}

Different EG samples were prepared via liquid phase exfoliation and were used to fabricate EDLCs in the form of EG/GPE/EG. In this study, EIS technique was performed to evaluate the electrochemical properties of the EDLCs. EIS test revealed the capacitive behavior of the EDLC through Nyquist and Bode plots. Thus the importance of using exfoliation and prior sonication as a low cost, environmental friendly method can be highlighted. Sri Lankan natural graphite showed better capacitance values than the commercially available graphite. Improvements may enhance the performances of Sri Lankan natural graphite based EDLCs and thus be a better solution for the demand of energy storage. 


\section{REFERENCES}

1. Yoo, H.D., Jang, J.H., Ryu, J.H., Park, Y.\& Oh, S.M., (2014). Impedance analysis of porous carbon electrodes to predict rate capability of electric double-layer capacitors. J Power Sources.267, pp.411-420. DOI: https://doi.org/10.1016/j.jpowsour.2014.05.058

2. Lei, C., Markoulidis, F., Ashitaka, Z. \& Lekakou, C., (2013). Reduction of porous carbon/Al contact resistance for an electric double-layer capacitor (EDLC). Electrochim Acta.92, pp.183-187.DOI:https://doi.org/10.1016/j.electacta.2012.12.092

3. Niu, L., Li, Z., Hong, W., Sun, J., Wang, Z., Ma, L., Wang, J. \& Yang, S., (2013). Pyrolytic synthesis of boron-doped graphene and its application aselectrode material for supercapacitors.Electrochim Acta.108, pp.666-673.

DOI: https://doi.org/10.1016/j.electacta.2013.07.025

4. Jung, M.J., Jeong, E., Lee, S.I. \& Lee, Y.S., (2012). Improved capacitance characteristics of activated carbon-based electrodes by physicochemical base-tuning. J Ind Eng Chem.18(2), pp.642-647. DOI: $\underline{\text { https://doi.org/10.1016/j.jiec.2011.11.055 }}$

5. Yamada, Y., Tanaka, T., MacHida K., Suematsu, S., Tamamitsu, K., Kataura, H. \& Hatori, H., (2012). Electrochemical behavior of metallic and semiconducting single-wall carbon nanotubes for electric double-layer capacitor. Carbon N Y.50(3), pp.1422-1424.

DOI: https://doi.org/10.1016/j.carbon.2011.09.062

6. Hao, P., Zhao, Z., Tian, J., Li, H., Sang, Y., Yu, G., Cai, H., Liu, H., Wong, C.P. \& Umar, A., (2014). Hierarchical porous carbon aerogel derived from bagasse for high performance supercapacitor electrode. Nanoscale.6(20), pp.12120-12129.

DOI: https://doi.org/10.1039/c4nr03574g

7. Cheng, Y., Huang, L., Xiao, X., Yao, B., Yuan, L., Li, T., Hu, Z., Wang, B., Wan, J. \& Zhou, J., (2015). Flexible and cross-linked N-doped carbon nanofiber network for high performance freestanding supercapacitor electrode. Nano Energy.15, pp.66-74.

DOI: https://doi.org/10.1016/j.nanoen.2015.04.007

8. Hantel, M.M., (2013). Graphite oxide and graphene oxide based electrode materials for electrochemical double layer capacitors.(21212), pp.1-340.

DOI: https://doi.org/10.3929/ethz-a-010782581

9. Iro, Z.S., Subramani, C. \& Dash, S.S., (2016). A brief review on electrode materials for supercapacitor. Int J Electrochem Sci.11(12), pp.10628-10643.

DOI: https://doi.org/10.20964/2016.12.50

10. Kakaei, K., Esrafili, M.D. \& Ehsani, A., (2019). Graphene-Based Electrochemical Supercapacitors. Interface Sci Technol.27(1), pp.339-386.

DOI: https://doi.org/10.1016/B978-0-12-814523-4.00009-5

11. Bhuyan, M.S.A., Uddin, M.N., Islam, M.M., Bipasha, F.A. \& Hossain, S.S., (2016). Synthesis of graphene. Int Nano Lett. 6(2), pp.65-83. DOI: https://doi.org/10.1007/s40089-015-0176-1

12. Narayan, R. \& Kim, S.O., (2015). Surfactant mediated liquid phase exfoliation of graphene. Nano Converg.2(1), pp.20-38. DOI:https://doi.org/10.1186/s40580-015-0050-x

13. Focke, W.W., Badenhorst, H., Ramjee, S., Kruger, H.J., Van Schalkwyk, R. \&Rand, B., (2014). Graphite foam from pitch and expandable graphite. Carbon $N$ Y.73, pp.41-50. DOI: https://doi.org/10.1016/j.carbon.2014.02.035 
14. Weerasinghe, W.A.D.S.S., Madhushani, L.K.M., Vidanapathirana, K.P. \&Perera, K.S., (2016). Performance of $\mathrm{Zn}$ rechargeable cells having Polypyrrole cathodes doped with surfactant anion. Ruhuna J Sci.6(2), pp.42-42.DOI: https://doi.org/10.4038/rjs.v6i2.10

15. Yang, I., Kim, S.G., Kwon, S.H., Kim, M.S. \& Jung, J.C., (2017). Relationships between pore size and charge transfer resistance of carbon aerogels for organic electric double-layer capacitor electrodes. Electrochim Acta.223, pp.21-30.

DOI: https://doi.org/10.1016/j.electacta.2016.11.177

16. Segalini, J., Daffos, B., Taberna, P.L., Gogotsi, Y. \& Simon, P., (2010). Qualitative Electrochemical Impedance Spectroscopy study of ion transport into sub-nanometer carbon pores in Electrochemical Double Layer Capacitor electrodes. Electrochim Acta. 55(25), pp.7489-7494. DOI:https://doi.org/10.1016/j.electacta.2010.01.003

17. Tey, J.P., Careem, M.A., Yarmo, M.A. \& Arof, A.K., (2016). Durian shell-based activated carbon electrode for EDLCs. Ionics (Kiel).22(7), pp.1209-1216.

DOI: https://doi.org/10.1007/s11581-016-1640-2

18. Wang, J., Chen, M., Wang, C., Wang, J. \& Zheng, J., (2011). Preparation of mesoporous carbons from amphiphilic carbonaceous material for high-performance electric double-layer capacitors.JPowerSources.196(1), pp.550-558.

DOI: https://doi.org/10.1016/j.jpowsour.2010.07.030 